\title{
Patient Characteristics Associated with Non-Adherence to Tuberculosis Treatment: A Systematic Review
}

\author{
A. 0. A. Ali1,2, Martin H. Prins ${ }^{2}$ \\ ${ }^{1}$ Ministry of Health, Saudi Arabia \\ ${ }^{2}$ Maastricht University Medical Centre, Maastricht, The Netherlands \\ Email: abuoosmann@Yahoo.com,mh.prins@maastrichtuniversity.nl
}

How to cite this paper: Ali, A.O.A. and Prins, M.H. (2020) Patient Characteristics Associated with Non-Adherence to Tuberculosis Treatment: A Systematic Review. Journal of Tuberculosis Research, 8, 73-92. https://doi.org/10.4236/jtr.2020.82008

Received: June 4, 2020

Accepted: June 27, 2020

Published: June 30, 2020

Copyright $\odot 2020$ by author(s) and Scientific Research Publishing Inc. This work is licensed under the Creative Commons Attribution International License (CC BY 4.0).

http://creativecommons.org/licenses/by/4.0/

\section{(c) (i) Open Access}

\begin{abstract}
Background: A high level of adherence to treatment is essential for cure and prevention of tuberculosis (TB) treatment resistance. Methods: A Systematic review of 53 studies addressing the patient characteristics associated with TB medication non-adherence was performed. The publications were identified by searching the PubMed, World Health Organization (WHO), and Centers for Disease Control and Prevention (CDC) database, EmBase, Scopus database Arts, Humanities, Social Science database and Google scholar. Only English language publications were eligible. Potentially eligible studies were retrieved and the full articles were assessed. The potentially eligible studies were included if they concerned patients treated for tuberculosis, reported non adherence and reported on potential risk factors associated with non-adherence. Results: Factors that were most frequently consistently and statistically significantly related to non-adherence to tuberculosis treatment were: family income, patient movement and changing address or giving wrong address, tuberculosis relapse or multidrug-resistant TB (MDRTB), during intensive phase of treatment, history of default, treatment regimen (long course), response to treatment, homeless, stigma, seeking traditional healers, staff receptiveness, lack of directly observed therapy short course (DOTS), poor knowledge or lack of health education, side effects of drugs, feeling better, alcohol intake and lack of family and social support. Conclusions: Non-adherence to tuberculosis treatment was influenced by several factors.
\end{abstract}

\section{Keywords}

Tuberculosis, Non-Adherence, Adherence, Defaulter, Compliance 


\section{Introduction}

Tuberculosis (TB) is among the top ten causes of global mortality [1] [2] [3] [4]. It is estimated that approximately one-third of the world's population (approximately two billion people) is infected with tuberculosis bacillus [5] [6] [7]. In 2015, globally 10.4 million new cases of TB and 1.8 million deaths from TB occurred, of which 1.4 million among HIV-negative people and 0.4 million deaths were in HIV-positive people and thus officially classified as HIV deaths in the International Statistical Classification of Diseases [8] [9] [10]. Approximately 80\% of TB cases are found in 22 countries; the highest incidence rates being found in Africa and South-East Asia [11].

TB has a high morbidity and mortality rate despite its status as a treatable disease [12] [13]. A high level of adherence to treatment is essential for cure and to avoid development of resistance [14] [15]. Thus, completion of antituberculosis treatment is the foremost priority of tuberculosis (TB) control programs. Treatment that is taken irregular, interrupted for two months or longer or is incomplete increases the risk of treatment failure, relapse of disease, acquisition of drug-resistant $\mathrm{TB}$, death, and prolonged infectiousness [16]-[21]. In turn, treatment failure and relapse can increase transmission of TB. Poor adherence to treatment is common despite various interventions aimed at improving treatment completion [22]. Non-adherence to TB medication is a major barrier to its local and global control and worsen the treatment outcome. In addition, defaulting increases the risk of drug resistance, relapse and death, and may prolong infectiousness. The objective of this systematic review was to identify the patient characteristics that are consistently associated with TB medication non-adherence. This knowledge could be helpful for health care providers and health policy makers to improve treatment adherence. The patient characteristics that we studied were: socio-demographic factors; disease related factors; treatment and services related factors; behavioral factors and social factors.

\section{Methods}

This systematic review was conducted to answer the question: which patient characteristics are consistently and statistically significantly associated with TB treatment non-adherence. The study reviewed publications found on risk factors associated with defaulting tuberculosis treatment that were published in English between 1990 to 2017. The publications were identified by searching the PubMed database, WHO database, and CDC database, EmBase, Scopus database, Arts, humanities, Social Science database and google scholar. Using the key words "tuberculosis", "mycobacterium tuberculosis", "adherence", "non adherence", "defaulting", "risk factors", "compliance", "determinant factors", "outcome of tuberculosis treatment", "predictors", "leading”, "impact”, "noncompliance”, “Anti tuberculosis treatment", and "anti tuberculosis therapy". Because of resource limitations, papers published in other languages were not considered. Additional reports were identified by manually reviewing the references of the studies found. 
The potentially eligible studies were included if the following criteria were all met: it concerned patients treated for tuberculosis (see definition below), it reported on adherence and non adherence, it reported on potential risk factors associated with adherence and non adherence, if the type of study is observation (cohort, case-control, cross-sectional), RCT and community survey, if it is a quantitative study or combined quantitative and qualitative, and if there is association ( $\mathrm{p}$ value, odds ratio or relative risk and $95 \%$ confidence interval, or $\mathrm{p}$ value, odds ratio or relative risk, or $\mathrm{p}$ value). The papers were excluded if they did not meet the above criteria, and if only an abstract was available. Selected articles were reviewed for information on the country of study, sample size, population source, type of the study, the statistical association, and risk factors e.g. age, sex, and other associated factors.

For purpose of data review simple definition were used according to those of the World Health organization publication (WHO, 2013) [23] [24].

A case of tuberculosis was defined as a patient in whom tuberculosis has been bacteriologically confirmed, or has been diagnosed by a clinician.

A bacteriologically confirmed TB case is one from whom a biological specimen is positive by smear microscopy, culture or WHO-approved rapid diagnostics (WRD) (such as Xpert MTB/RIF). All such cases should be notified, regardless of whether TB treatment has started.

A clinically diagnosed TB case is one who does not fulfil the criteria for bacteriological confirmation but has been diagnosed with active TB by a clinician or other medical practitioner who has decided to give the patient a full course of TB treatment. This definition includes cases diagnosed on the basis of X-ray abnormalities or suggestive histology and extrapulmonary cases without laboratory confirmation. Clinically diagnosed cases subsequently found to be bacteriologically positive (before or after starting treatment) should be reclassified as bacteriologically confirmed.

Pulmonary tuberculosis (PTB) refers to any bacteriologically confirmed or clinically diagnosed case of $\mathrm{TB}$ involving the lung parenchyma or the tracheobronchial tree. Miliary TB is classified as PTB because there are lesions in the lungs. Tuberculous intra-thoracic lymphadenopathy (mediastinal and/or hilar) or tuberculous pleural effusion, without radiographic abnormalities in the lungs, constitutes a case of extrapulmonary TB. A patient with both pulmonary and extrapulmonary TB should be classified as a case of PTB. Extrapulmonary tuberculosis (EPTB) refers to any bacteriologically confirmed or clinically diagnosed case of TB involving organs other than the lungs, e.g. pleura, lymph nodes, abdomen, genitourinary tract, skin, joints and bones, meninges.

Potentially eligible studies were excluded if they did not report on potential risk factors associated with adherence and non adherence, if no full articles available or if the review. Potentially eligible studies were retrieved and the full articles were assessed. Results are reported according to the PRISMA guidelines for systematic reviews [25]. 
Data was extracted by A.A.Ali. For the purpose of data extraction a simple definition for treatment adherence and nonadherence was used according to that of the World Health organization publication (WHO, 2002) [22] [26]. Non-adherence (or Defaulter): A patient whose treatment was interrupted for 2 or more consecutive months.

The following five categories of risk factors for TB treatment non-adherence were considered:

1) Sociodemogaphic factors including: age, sex, ethnicity, marital status, educational level, occupation, employment status, family income, nationality, and residential locality, religion, patients moving or changing address, family size and house size [11] [27]-[40].

2) Disease related factors including: type of tuberculosis (first time to develop tuberculosis, relapse, multi-drug resistant tuberculosis), sputum smear result, severity of disease, and site of tuberculosis [41]-[51].

3) The treatment and services related factors including: treatment regimen, time of defaulting, directly observation therapy short course (DOTS), drugs availability, drug cost, response to treatment, side effects of drugs, distance of residence from treatment center, waiting time, travelling time, travelling cost, satisfaction with treatment and services, staff receptiveness towards the patients, history of default, treatment (in patient or out-patient), type of clinic (governmental or private), hospital referral, and difficulties facing tracing system for defaults [31] [42] [52]-[61].

4) Selected behavioral factors including: smoking, alcohol intake, IVDU and HIV status, homelessness, prison and immigrant [19] [45] [62] [63] [64] [65] [66].

5) other factors influencing defaulting including; presence or absence of social support such as family support, health education and patient knowledge, stigma, chronic diseases, traditional treatment, feeling better, and need incentive [13] [67]-[76].

When possible we extracted odds ratios (ORs), relative risks (RRs), and their 95\% confidence interval. Otherwise the direction of the association and statistical significance was used. The relationship was considered consistent if the variable was reported in 3 studies or more to be statistically significantly related with non-adherence to tuberculosis treatment and more often statistically significantly related to non-adherence than none related. Ethics approval was not required for this systematic review.

\section{Results}

The database searching resulted in a total of 2131 identified citations. About 981 records remained after removal of duplication. Out of these 894 were excluded because they did not focus on the TB treatment and risk factors. Of the $87 \mathrm{ab}$ stracts that were potentially eligible, 34 were excluded after the review of the full paper or abstract (see Figure 1). Hence 53 eligible articles were finally included. 


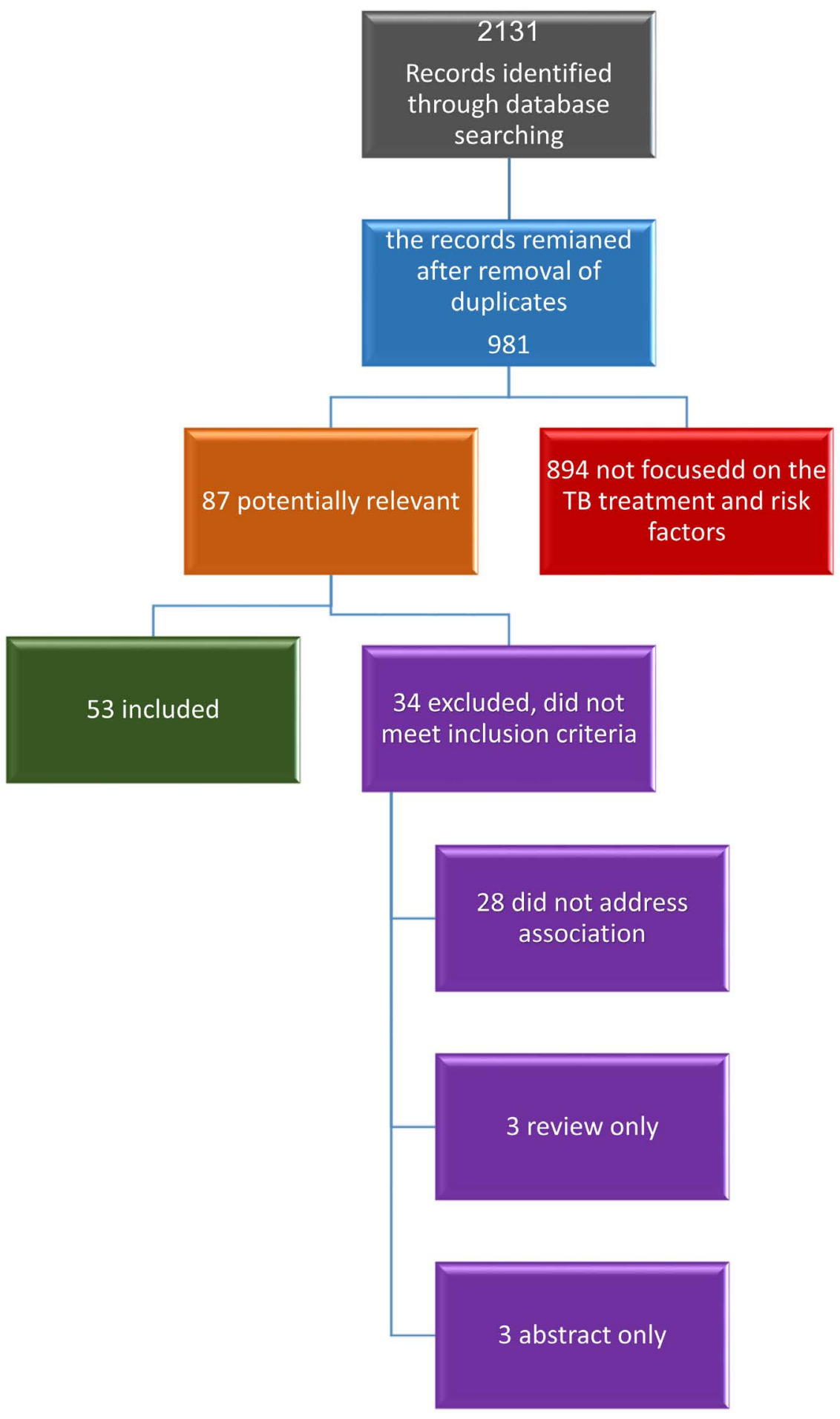

Figure 1. Study flow diagram.

Of these 53 eligible studies, 21 were conducted in Africa, 19 in Asia, five in North America, four in Europe and four in South America.

The study design included 26 cohort studies, 17 case control studies, eight cross-sectional studies, and two surveys. The main characteristics of each study are reported in Table 1. 
Table 1. The design of the studies (patient characteristics associated with non-adherence to tuberculosis treatment).

\begin{tabular}{|c|c|c|c|c|c|c|}
\hline NO & Author & $\begin{array}{l}\text { Country and } \\
\text { area }\end{array}$ & $\mathbf{N}$ & $\begin{array}{l}\text { Default } \\
\text { rate }\end{array}$ & Study type & Population \\
\hline 1 & $\begin{array}{l}\text { VAN DER Werf T S (1990) } \\
\qquad[15]\end{array}$ & Ghana & 569 & 10 & $\begin{array}{l}\text { Retrospective } \\
\text { cohort study }\end{array}$ & $\begin{array}{l}\text { Sputum-smear positive pulmonary tuberculosis } \\
\text { (PTB) patients registered between } 1984 \text { and } 1987 \text { in } \\
\text { a rural ambulatory non-supervised service program } \\
\text { in Agogo Hospital in the hills of Ashanti, Ghana }\end{array}$ \\
\hline 2 & Chuah S Y (1991) [17] & Malaysia & 227 & 33 & $\begin{array}{l}\text { Retrospective } \\
\text { cohort study }\end{array}$ & $\begin{array}{l}\text { Tuberculosis patients in Perak started treatment in } \\
1982\end{array}$ \\
\hline 3 & Menzies R (1993) [18] & Canada & 352 & 16 & $\begin{array}{l}\text { Retrospective } \\
\text { cohort study }\end{array}$ & $\begin{array}{l}\text { Adult Tuberculosis patients treated at the tuberculosis } \\
\text { clinic of Montreal Chest Hospital in 1987-1988 }\end{array}$ \\
\hline 4 & Me'ndez A P (1997) [66] & USA & 184 & 48 & $\begin{array}{l}\text { Retrospective } \\
\text { cohort study }\end{array}$ & $\begin{array}{l}\text { All patients in New York City with a first-time } \\
\text { positive culture for Mycibacterium tuberculosis in } \\
\text { April } 1991 \text { were selected }\end{array}$ \\
\hline 5 & $\begin{array}{l}\text { Ngamvithayapong J (1997) } \\
\qquad[22]\end{array}$ & Thailand & 412 & 26 & $\begin{array}{l}\text { Prospective } \\
\text { cohort study }\end{array}$ & $\begin{array}{l}\text { 1) blood donors who had indicated a desire to know } \\
\text { their HIV test result; 2) persons attending the } \\
\text { hospital's anonymous counselling and testing clinic; } \\
\text { 3) patients attending the hospital's outpatient clinics } \\
\text { for other illnesses but found to be HIV-infected, and } \\
\text { 4) female commercial sex workers }\end{array}$ \\
\hline 6 & Jochem K (1997) [24] & Nepal & 693 & 14.8 & $\begin{array}{l}\text { Prospective } \\
\text { cohort study }\end{array}$ & New cases of Smear positive tuberculosis patients \\
\hline 7 & William J (1997) [20] & USA & 294 & 18 & $\begin{array}{l}\text { Retrospective } \\
\text { cohort study }\end{array}$ & $\begin{array}{l}\text { All patients treated with outpatient DOT from } 1984 \\
\text { to } 1994\end{array}$ \\
\hline 8 & KATE C (1998) [67] & USA & 2576 & 5.5 & Cohort study & $\begin{array}{l}\text { Adult patients at least ( } 15 \mathrm{yr} \text { of age) reportedas } \\
\text { having TB in California during } 1993\end{array}$ \\
\hline 9 & Comolet T M (1998) [27] & Madagascar & 149 & 25.5 & Case-control study & Sputum positive tuberculosis patient \\
\hline 10 & Tanguis H G (2000) [62] & Spain & 2201 & 13.1 & Cohort study & HIV-infected with TB patients between 1987 and 1996 \\
\hline 11 & $\begin{array}{l}\text { Borgdorff M W J } \\
\quad(2000)[63]\end{array}$ & Netherland & 7529 & 10 & Cohort study & $\begin{array}{l}\text { Tuberculosis patient in the Netherlands in the period } \\
1993-1997\end{array}$ \\
\hline 12 & El-Sony A I (2002) [13] & Sudan & 1797 & 16.9 & Cross-sectional study & $\begin{array}{l}\text { All persons aged } 15 \text { - } 49 \text { years with respiratory } \\
\text { symptoms seen in the general health services from } \\
\text { March } 1998 \text { to March 2000,from eight states } \\
\text { in Sudan }\end{array}$ \\
\hline 13 & Tekle B (2002) [76] & Ethiopia & 1367 & 11.3 & A case control study & New tuberculosis patients on DOTS \\
\hline 14 & Edginton M E (2002) [29] & South Africa & 303 & 5.6 & A descriptive study & $\begin{array}{l}\text { TB patients and community members in the rural } \\
\text { Tintswalo district of the Northern Province of South } \\
\text { Africa registered with tuberculosis in the 18-month } \\
\text { period }\end{array}$ \\
\hline 15 & Santha T (2002) [41] & India & 676 & 17 & A community survey & $\begin{array}{l}\text { All tuberculosis patients registered from May } 1999 \\
\text { through April } 2000\end{array}$ \\
\hline 16 & Chan-Yeung M (2003) [53] & $\begin{array}{l}\text { China, } \\
\text { Hong Kong }\end{array}$ & 5757 & 8 & $\begin{array}{l}\text { Nested case-control } \\
\text { study }\end{array}$ & $\begin{array}{l}\text { Patients registered for antituberculous drug therapy } \\
\text { in } 1996 \text { in Hong Kong }\end{array}$ \\
\hline 17 & Sophia V (2003) [54] & India & 483 & 25 & Case control study & $\begin{array}{l}\text { New and re-treatment smear positive patients } \\
\text { followed up till treatment outcome from March to } \\
\text { December } 1999 \text { and March } 1999 \text { to September } 2000\end{array}$ \\
\hline 18 & Robert M (2004) [21] & USA & 372 & 12.6 & $\begin{array}{l}\text { Prospective } \\
\text { cohort study }\end{array}$ & $\begin{array}{l}\text { Culture-positive patients who were reported from } \\
1998-2000\end{array}$ \\
\hline 19 & Chang K C (2004) [31] & $\begin{array}{l}\text { China, } \\
\text { Hong Kong }\end{array}$ & 408 & 8.5 & $\begin{array}{l}\text { Nested case-control } \\
\text { study }\end{array}$ & $\begin{array}{l}\text { All tuberculosis patients registered at the Government } \\
\text { chest clinics from } 1 \text { January } 1999 \text { to } 31 \text { March } 1999\end{array}$ \\
\hline 20 & Hill P C (2005) [42] & Gambia & 301 & 25.2 & $\begin{array}{l}\text { Prospective } \\
\text { cohort study }\end{array}$ & $\begin{array}{l}\text { New cases of TB aged }>15 \text { years who started their } \\
\text { treatment between } 16 \text { October } 2002 \text { and } \\
26 \text { November } 2003\end{array}$ \\
\hline
\end{tabular}




\section{Continued}

\begin{tabular}{|c|c|c|c|c|c|c|}
\hline 21 & Pandit N S K (2006) [70] & India & 274 & 6.2 & $\begin{array}{l}\text { Cross sectional } \\
\text { study }\end{array}$ & $\begin{array}{l}\text { All the patients who registered for DOT treatment } \\
\text { during last quarter (Oct-Nov-Dec) 2002, in Anand } \\
\text { district, state Gujarat, India }\end{array}$ \\
\hline 22 & Daniel O J (2006) [64] & Nigeria & 774 & 23 & $\begin{array}{l}\text { Retrospective } \\
\text { cohort study }\end{array}$ & $\begin{array}{l}\text { Adult Tuberculosis patients age } 15 \text { years and above } \\
\text { registered for anti-TB treatment DOTS between } \\
\text { January } 1997-D e c e m b e r ~ 2003 \text {, Sagamu, Nigeria }\end{array}$ \\
\hline 23 & Estifanos B S (2007) [32] & Ethiopia & 404 & 20 & $\begin{array}{l}\text { Prospective } \\
\text { cohort study }\end{array}$ & $\begin{array}{l}\text { Smear-positive tuberculosis patients in } \\
\text { Hossana Hospital }\end{array}$ \\
\hline 24 & Gelmanova I Y (2007) [43] & Russia & 237 & 8.8 & $\begin{array}{l}\text { Retrospective } \\
\text { cohort study }\end{array}$ & $\begin{array}{l}\text { Detected, smear and/or culture-positive adult } \\
\text { TB patients initiating therapy in a DOTS } \\
\text { programme in Tomsk between } 1 \text { January } \\
\text { and } 31 \text { December } 2001\end{array}$ \\
\hline 25 & Fahrettin T (2008) [44] & Turkey & 586 & 5.1 & $\begin{array}{l}\text { Retrospective } \\
\text { cohort study }\end{array}$ & $\begin{array}{l}\text { Adult pulmonary tuberculosis patient age } \\
\text { more than } 15 \text { years in Istanbul Eyup } \\
\text { tuberculosis Dispensary between year } \\
1999-2004\end{array}$ \\
\hline 26 & Franke M F (2008) [50] & Peru & 671 & 10 & Retrospective cohort & Patients with multi drug resistant $\mathrm{TB}$ disease \\
\hline 27 & Kapella B K (2009) [71] & Thailand & 995 & 40 & Cohort study & $\begin{array}{l}\text { Registered TB treatment (non Thai) from } \\
1 \text { October } 2004 \text { to } 31 \text { September } 2006 .\end{array}$ \\
\hline 28 & Xu L (2010) [33] & China & 501 & 9 & $\begin{array}{l}\text { A patient-based } \\
\text { study survey }\end{array}$ & $\begin{array}{l}\text { Rural smear-positive pulmonary TB patients } \\
\text { registered with the county TB dispensaries at study } \\
\text { sites who completed treatment during the period } \\
\text { October } 2006 \text { to September } 2007 \text {. }\end{array}$ \\
\hline 29 & Kliiman K (2010) [65] & Estonia & 1107 & 9.4 & $\begin{array}{l}\text { A retrospective } \\
\text { cohort study }\end{array}$ & $\begin{array}{l}\text { patients with culture-confirmed pulmonary TB } \\
\text { who started treatment during 2003-2005 }\end{array}$ \\
\hline 30 & Samuel A B (2010) [72] & Ghana & 165 & 55.7 & Cross-sectional study & $\begin{array}{l}\text { TB patients who had registered in New Juaben } \\
\text { District from January } 2003 \text { to December } 2005 \\
\text { (TB Registry) and had fully completed their } \\
\text { treatment during the time of the study }\end{array}$ \\
\hline 31 & Muture B N (2011) [45] & Kenya & 1978 & 16.7 & A Case-Control study & $\begin{array}{l}\text { TB patients (adults and children) registered } \\
\text { during the period January } 2005 \text { to March } 2007\end{array}$ \\
\hline 32 & Kelly E D (2011) [51] & Morocco & 291 & 13 & $\begin{array}{l}\text { A retrospective } \\
\text { cohort study }\end{array}$ & $\begin{array}{l}\text { Patients with smear- or culture-positive } \\
\text { pulmonary tuberculosis }\end{array}$ \\
\hline 33 & Finlay A (2012) [46] & South Africa & 3165 & 12 & $\begin{array}{l}\text { retrospective case } \\
\text { control study }\end{array}$ & $\begin{array}{l}\text { Adult persons } \geq 18 \text { years old enrolled in treatment } \\
\text { under DOTS at public health facilities in South } \\
\text { Africa between January } 1 \text { and December } 31,2002\end{array}$ \\
\hline 34 & Culqui D R (2012) [56] & Peru & 789 & 5 & $\begin{array}{l}\text { An unmatched- } \\
\text { case-control study }\end{array}$ & $\begin{array}{l}\text { Patients diagnosed with tuberculosis from } 2004 \text { to } \\
2005 \text { who completed treatment until September } 2006 \\
\text { in Peru }\end{array}$ \\
\hline 35 & Garrido M D S (2012) [60] & Brazilia & 11,321 & 10 & Case control study & TB patients from 2005-2010 in the Amazonas State \\
\hline 36 & Tamhane A (2012) [75] & India & 150 & 29 & Cross-sectional study & $\begin{array}{l}\text { Newly diagnosed sputum smear-positive pulmonary } \\
\text { tuberculosis (TB) patients }\end{array}$ \\
\hline 37 & Nezenega Z S (2013) [61] & Ethiopia & 531 & 26 & Cross sectional study & $\begin{array}{l}\text { TB patients on anti TB treatment in } 11 \text { public } \\
\text { health centers and one hospital of Sidama zone }\end{array}$ \\
\hline 38 & Lalor M K (2013) [49] & Uzbekistan & 710 & 20 & A retrospective cohort & $\begin{array}{l}\text { Multi and extensively drug-resistant tuberculosis } \\
\text { patients who started treatment between } 2003 \text { and } \\
2008 \text { and thus had finished approximately } 2 \text { years } \\
\text { of treatment by the end of } 2010\end{array}$ \\
\hline 39 & Slama K (2013) [39] & Morocco & 320 & 33.8 & Case-control study & $\begin{array}{l}\text { TB patients (new cases or relapsed) who were } \\
\text { enrolled in treatment for TB between } 1 \text { January } 2009 \\
\text { and } 31 \text { December } 2010\end{array}$ \\
\hline
\end{tabular}




\section{Continued}

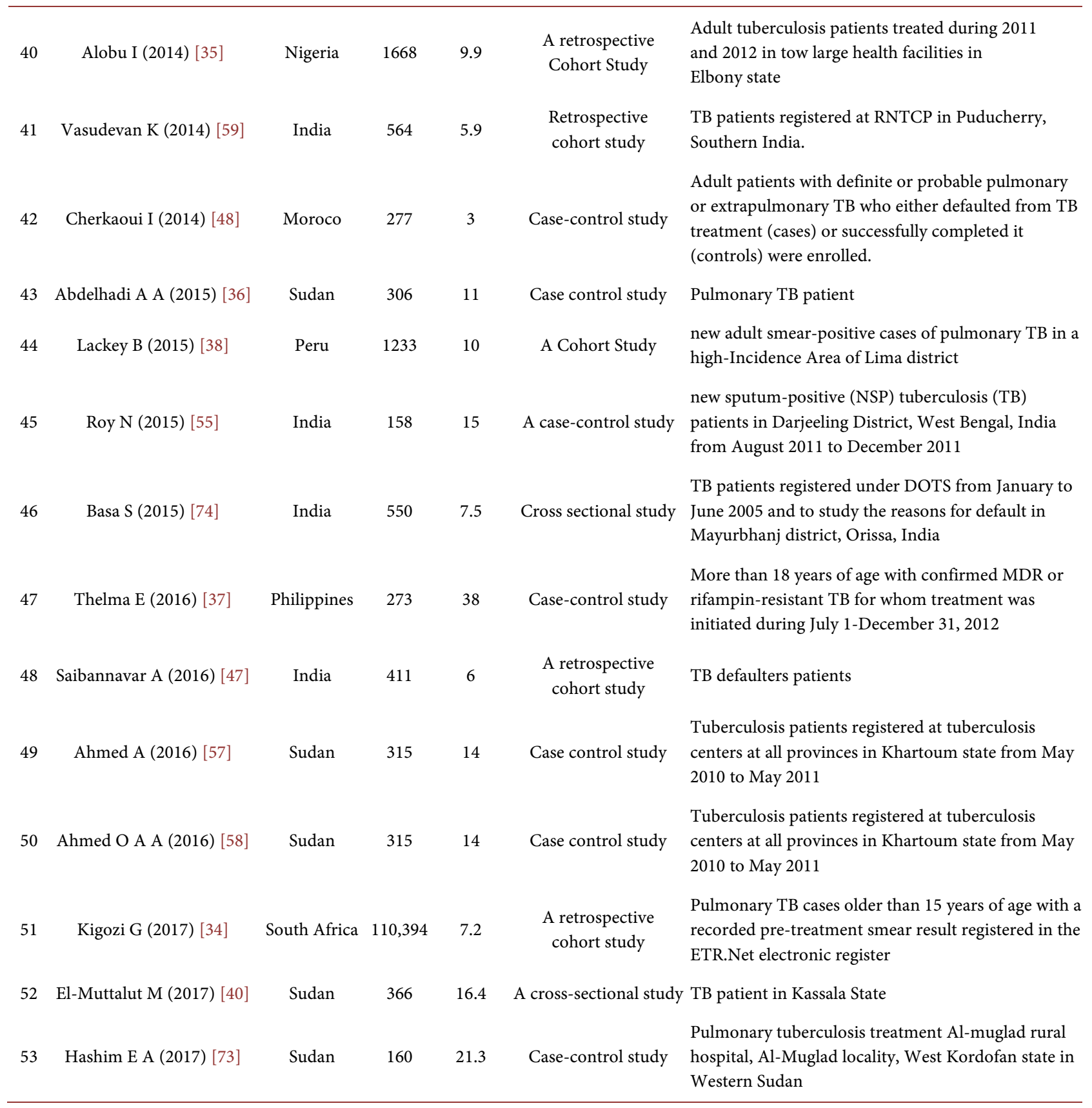

The factors associated with non-adherence to tuberculosis treatment are given in Tables 2(a)-(e). Factors that were most frequently, consistently and statistically significantly related to tuberculosis treatment non-adherence were; low family income reported odds ratio (OR: 1.61 to 11.24), patient moving or giving wrong address reported relative risk (RR: 2.36 to 5.5), tuberculosis relapse or multidrug resistant (MDR) TB (OR 1.61 to 12), intensive phase of treatment (OR: 1.78 to 117.21), history of default (OR 1.09 to 8.2), long course treatment regimen (OR: 1.096 to 6.9), response to treatment (OR: 2.4 to 9.9), side effect of drugs (OR: 1.1 to 13.3), alcohol intake (OR: 1.3 to 4.9 ) homeless (OR: 2.0 to 3.2), 
Table 2. (a) Socio-demographic factors consistently associated with non-adherence; (b) Behavioral factors consistently associated with non-adherence; (c) Treatment and services related factors consistently associated with non-adherence; (d) Disease related factors consistently associated with non-adherence; (e) Other (miscellaneous) factors consistently associated with non-adherence.

(a)

\begin{tabular}{|c|c|c|c|c|}
\hline No. & $\begin{array}{l}\text { Socio-demographic } \\
\text { factors }\end{array}$ & $\begin{array}{c}\text { Factors associated \& } \\
\text { statistically } \\
\text { significant }\end{array}$ & $\begin{array}{c}\text { Factors associated } \\
\text { but not statistically } \\
\text { significant }\end{array}$ & Factors not associated \\
\hline 1 & Age & $\begin{array}{c}15,32,34,71,35,34 \\
36,47,74,48,49,60\end{array}$ & $\begin{array}{l}67,41,54,42 \\
46,37,38,40\end{array}$ & $\begin{array}{c}17,18,22,20,27,62,63,53, \\
31,70,64,43,33,72,45,39 \\
73,55,56,57,58,50,51,65 \\
75,66\end{array}$ \\
\hline 2 & Gender & $\begin{array}{c}15,17,22,27,41,53, \\
54,64,72,45,34,47, \\
74,56,51\end{array}$ & $\begin{array}{c}20,63,44,46 \\
38,48,75\end{array}$ & $\begin{array}{c}18,62,31,42,32,43,71,33 \\
37,39,40,49,55,57,58,50 \\
60,65,66\end{array}$ \\
\hline 3 & Ethnicity & 47 & $20,63,54,66$ & $17,31,42,72,46$ \\
\hline 4 & Marital status & 22,47 & 45 & $\begin{array}{c}15,31,32,44,71,33,46,36 \\
38,39,40,73,49,55,56,57 \\
51,75\end{array}$ \\
\hline 5 & Educational level & $46,36,38,40,47,60$ & $\begin{array}{c}15,27,45,48 \\
57,50,75\end{array}$ & $\begin{array}{c}22,41,54,70,32,44,33,72 \\
39,55,56,65,66,76\end{array}$ \\
\hline 6 & Occupation & $17,47,48,57$ & 72 & $\begin{array}{c}69,41,31,42,32,71,33,36 \\
37,40\end{array}$ \\
\hline 7 & Employment status & $73,74,65$ & 48,75 & $54,43,45,46,39,49,55,76$ \\
\hline 8 & Family income & $\begin{array}{c}62,33,72,45,40,73 \\
47,56\end{array}$ & $54,39,48,66$ & $32,37,57,75,76$ \\
\hline 9 & Nationality, & 63 & 20,46 & $18,36,57$ \\
\hline 10 & Residential locality & $27,40,57,58,50,65$ & $20,45,35,34,37$ & $17,63,31,32,71,48,60$ \\
\hline 11 & Religion & 59 & & $27,72,46,47,56,57$ \\
\hline 12 & $\begin{array}{l}\text { Patients moving or } \\
\text { changing address }\end{array}$ & $\begin{array}{c}67,27,54,71,45,46 \\
73,57\end{array}$ & 49,75 & 55 \\
\hline 13 & Family size & & 48 & $45,57,75$ \\
\hline 14 & House size & 50 & & $45,39,57$ \\
\hline
\end{tabular}

(b)

\begin{tabular}{ccccc}
\hline No. & Behavioral factors & $\begin{array}{c}\text { Factors associated \& } \\
\text { statistically significant }\end{array}$ & $\begin{array}{c}\text { Factors associated but not } \\
\text { statistically significant }\end{array}$ & $\begin{array}{c}\text { Factors not } \\
\text { associated }\end{array}$ \\
\hline 1 & Smoking & $31,39,48$ & $41,37,38,55$ & $49,58,51,75$ \\
2 & Alcohol intake & $\begin{array}{c}20,67,41,45,46,37,39, \\
73,74,55,60,65\end{array}$ & 43,66 & $27,62,31,49,58$, \\
3 & IVDU & $67,43,38,56,50,66$ & 51 & 51,65 \\
4 & HIV status & $45,34,38,60$ & $67,62,31,64,66$ & $20,63,13,71,58$ \\
5 & Homelessness & $20,67,62,63,66$ & 65 & \\
6 & Prison & $67,63,65$ & 62,38 & $49,38,71$ \\
7 & Immigrant & 63,74 & 22 & \\
\hline
\end{tabular}


(c)

\begin{tabular}{|c|c|c|c|c|}
\hline No. & Treatment and services related & $\begin{array}{l}\text { Factors associated \& } \\
\text { statistically significant }\end{array}$ & $\begin{array}{l}\text { Factors associated } \\
\text { but not statistically } \\
\text { significant }\end{array}$ & $\begin{array}{l}\text { Factors not } \\
\text { associated }\end{array}$ \\
\hline 1 & $\begin{array}{l}\text { Treatment regimen } \\
\text { (long course) }\end{array}$ & $\begin{array}{c}18,24,13,54,72,35,74 \\
49,59,50,66\end{array}$ & & $17,67,71,47$ \\
\hline 2 & $\begin{array}{l}\text { Time of defaulting (intensive } \\
\text { phase or continuation phase }\end{array}$ & $\begin{array}{c}17,18,53,54,31,42,64 \\
45,36,59,61,76\end{array}$ & $41,37,48$ & \\
\hline 3 & DOTS & $\begin{array}{c}15,62,21,70,53,33,35 \\
74,48,60,66\end{array}$ & & 31 \\
\hline 4 & Drugs availability & 29,61 & 72 & \\
\hline 5 & Drug cost & 68,75 & & 70 \\
\hline 6 & Response to treatment & $24,20,29,54,42,43,66$ & 50 & \\
\hline 7 & Side effects of drug & $\begin{array}{c}29,31,70,43,72,37,74 \\
48,55,56,76\end{array}$ & $22,54,46,45$ & $15,20,27,39$ \\
\hline 8 & $\begin{array}{l}\text { Distance of residence } \\
\text { from treatment center }\end{array}$ & $15,29,32,33,72,47,74$ & 45,57 & $\begin{array}{l}17,22,54,37 \\
73,48,50,76\end{array}$ \\
\hline 9 & Waiting time & 29,61 & 45 & $70,46,58$ \\
\hline 10 & Travelling time & $27,42,39$ & 37,57 & $15,70,43,73,75$ \\
\hline 11 & Travelling cost & 29,42 & 32,57 & 15,70 \\
\hline 12 & $\begin{array}{l}\text { Satisfaction with } \\
\text { treatment and services }\end{array}$ & 73,61 & & 58 \\
\hline 13 & Staff receptiveness & $\begin{array}{c}18,27,29,33,72,46,37 \\
73,74,55,58,75,61\end{array}$ & 45 & \\
\hline 14 & History of default & $\begin{array}{c}18,41,53,54,31,45,46 \\
70,71,72,73,76\end{array}$ & 51 & 65 \\
\hline 15 & $\begin{array}{c}\text { Treatment } \\
\text { (in patient or out-patient) }\end{array}$ & 22,31 & & 46 \\
\hline 16 & $\begin{array}{c}\text { Type of clinic } \\
\text { (governmental or private) }\end{array}$ & & 35 & \\
\hline 17 & Hospital referral & 17 & & \\
\hline 18 & $\begin{array}{l}\text { Difficulties facing tracing } \\
\text { system for defaults }\end{array}$ & 55 & & \\
\hline 19 & $\begin{array}{l}\text { Access health-care services } \\
\text { within office hours }\end{array}$ & 56 & & \\
\hline 20 & $\begin{array}{l}\text { Too many patients when } \\
\text { visiting health center }\end{array}$ & 58 & & \\
\hline 22 & Health center appearance & 58 & & 76 \\
\hline
\end{tabular}

(d)

\begin{tabular}{|c|c|c|c|c|}
\hline No. & Disease related factors & $\begin{array}{c}\text { Factors associated } \\
\& \text { statistically } \\
\text { significant }\end{array}$ & $\begin{array}{c}\text { Factors associated } \\
\text { but not statistically } \\
\text { significant }\end{array}$ & Factors not associated \\
\hline 1 & $\begin{array}{ll} & \text { Type of tuberculosis } \\
\text { - } & \text { first time to develop } \\
\text { - } & \text { relapse } \\
\text { - } & \text { multi-drug resistant }\end{array}$ & $\begin{array}{c}67,62,41,53,43 \\
44,46,34,38,39 \\
49,65,66\end{array}$ & & $63,71,45,37$ \\
\hline 2 & Severity of disease & 22,51 & & $15,17,53,66$ \\
\hline
\end{tabular}




\section{Continued}

\begin{tabular}{ccccc}
\hline 3 & Sputum smear result & $18,24,35,50,51$ & 63,34 & $15,17,13,53,31,64,43$, \\
& & $37,60,65,66$ \\
4 & Site of tuberculosis & 35 & $17,41,48,57$ & $53,44,39,66$ \\
\hline
\end{tabular}

(e)

\begin{tabular}{ccccc}
\hline No. & $\begin{array}{c}\text { Other factors } \\
\text { influencing defaulting }\end{array}$ & $\begin{array}{c}\text { Factors associated \& } \\
\text { statistically significant }\end{array}$ & $\begin{array}{c}\text { Factors associated but not } \\
\text { statistically significant }\end{array}$ & $\begin{array}{c}\text { Factors not } \\
\text { associated }\end{array}$ \\
\hline 1 & Social support & $29,72,37,74,48,57,58,76$ & & \\
& $\begin{array}{c}\text { Health education, } \\
\text { counseling and patient } \\
\text { knowledge }\end{array}$ & $\begin{array}{c}18,27,29,54,70,72,45, \\
46,39,48,55,56,58,\end{array}$ & 42 & 36 \\
7 & Stigma & $22,29,70,72,46,36,73$ & 45,58 & $31,71,48$ \\
4 & Chronic diseases & 53,60 & 38,50 & \\
5 & Traditional treatment & $72,45,46,74,75$ & 41 & \\
6 & Feeling better & $46,36,39,40,74,48$ & 37 & \\
7 & Need incentive & 46 & 37 & \\
\hline
\end{tabular}

stigma (OR: 2.0 to 5.0), seeking traditional healers (OR: 1.9 to 5.7), staff receptiveness (OR: 1.7 to 12.3), lack of DOTS (OR: 1.42 to 2.29), poor knowledge or lack of health education (OR: 1.88 to 8.67), lack family and social support (OR: 2.14 to 3.23 ) and feeling better (OR: 5.28 to 21.0 ).

Factors frequently but not consistently related to tuberculosis treatment nonadherence were: male, residential locality, distance to tuberculosis treatment unit, HIV status, IVDU, prison, and smoking.

All studies reported the default rates. The reported default rates ranged between $3 \%$ and $55.7 \%$. These default rates varied substantially among the studies as either cumulative or incremental percentages of all patients. Also it varied according to regimen of treatment, age, sex, ethnicity, and residential locality.

All the papers included in the systematic synthesis had reported the number of patients (the sample size) participated in the individual study. The total number of the participant subjects in all articles was 165,842 .

Ninety eight percent of the studies included in the systematic review showed a significant effect of the 17 related factors on TB treatment default.

The number of studies showed increased risk of TB treatment default for each of the 17 significant variables varied from 5 to 16 studies: $5 / 52$ for homeless and seeking traditional healers; $6 / 52$ for feeling better; $7 / 52$ for response to treatment and stigma; 8/52 for low family income, patient moving or giving wrong address, and lack of family and social support ; 11/52 for long course treatment regimen, lack of DOTS, and side effects of drugs; $12 / 52$ for intensive phase of treatment and alcohol intake; 13/52 for tuberculosis relapse or multidrug resistant (MDR) TB, staff receptiveness and history of default; and 16/52 for poor knowledge or lack of health education and counseling. 


\section{Discussion}

The results of this systematic review show that family income, moving of patient or giving wrong address, tuberculosis relapse or MDR TB, intensive phase of treatment, history of default, long course treatment regimen, response to treatment, homeless, stigma, seeking traditional healers, staff receptiveness, DOTS, poor knowledge or lack of health education were consistently and statistically significantly related to tuberculosis treatment non-adherence. Surprisingly some frequently cited factors which were traditionally thought to be related to tuberculosis treatment non-adherence appeared to be not consistently associated with non-adherence, these include age, sex, marital status, occupation, employment status, level of education.

The most important methodological aspect of this systematic review that requires explanation is the use of a qualitative approach, i.e. counting statistical results of the patient characteristics related to non-adherence to tuberculosis treatment. Ideally, numerical results of individual studies are combined. However, when reviewing eligible studies it was discovered that in most of these primary studies only numerators were given if a factor was statistically significant related to non-adherence. Hence, only including the results of a characteristic if full numerical data was given had the potential to lead to an extreme bias. Since studies dating back to 1990 were included we assumed that it was unlikely to get full responses of all authors. Hence, we resorted to, admittedly a less optimal qualitative approach.

Some other methodological aspects of this systematic review require attention: First of all, we attempted to identify all relevant articles published up to date of this review and found a large number of relevant references. However, due to language restriction and lack of resources we only included articles in the English language. Secondly, data extraction was hampered by unclear methodology and definitions in many relevant studies. In addition, many relevant factors for non-adherence were not taken into account in many studies. Thirdly, the majority of articles included in this study were conducted in developing countries; the findings are therefore most applicable to countries with low resources which carry the greatest burden of TB disease and where urgent interventions are needed to improve adherence to tuberculosis treatment. However, our findings may also be applicable to more developed countries. Fourthly, our review took many types of risk factors (social and biomedical) for non-adherence to $\mathrm{Tb}$ treatment into account while many individual articles solely have focused on the factors related to health services provision.

Overall we trust that the factors identified as consistently related to TB treatment non-adherence are of truly associated with non-adherence. For the potential risk factors for non-adherence that were not consistently associated a numerical approach in large data set of new studies might still show an association. However, it is likely that these associations are of little importance.

Exploration of the factors associated with non-adherence to tuberculosis treatment deserves some attention. Some of these factors identified can be changed and 
solved. These include factors associated with patient behavior: moving of patient or giving wrong address, homeless, stigma and seeking traditional healers, and factors related to treatment services: not on DOTS, long course treatment regimen, staff receptiveness and poor knowledge or lack of health education. Other factors related to non-adherence are difficult to change as they are not of patients or the treating physician control. Most of these factors are related to the treatment and the system of services provision and include: low family income, tuberculosis relapse or MDR TB, intensive phase of treatment, history of default and response to treatment. Hence, the presence of these factors might guide to the targeting of extra attempts to stimulate treatment adherence.

The doctor-patient-relationship offers good environment for communication giving patients comfort in expressing the obstacles they face during treatment course. This enables treating doctors and health worker staff to analyze patients' problems and provide advice, counseling and health education regarding type of disease, side effect of drug, duration of treatment, implementation of DOT short course program. Moreover, it will enable health care workers to be more flexible with patients in giving them choices and options that maintain their autonomy in treatment compliance and to overcome obstacles related to health services provision.

The doctors and health worker staff receptiveness and their effort in counseling and health education provision increases awareness of patients and their families and the whole community about Tuberculosis. The social support positively affects the patients' knowledge to counteract the feeling of guilt and shame (stigma). This will be reflected on patients' compliance with tuberculosis medication instead of seeking traditional medicine. Patients' compliance assists the tuberculosis control program to achieve high cure rates and decrease the MDR prevalence in the community.

This synthesis suggests that governmental and nongovernmental organizations need to give more attention to support poor patients financially and offer accommodation for the homeless. Another cost effective suggestion is to consider even distribution of health services to improve its availability, accessibility and affordability to all tuberculosis patients. This, combined with good referral system from health unit to hospital, will result in reduction of the cost of travelling, waiting time at tuberculosis clinic, and patient movement. Besides, good counseling to patients would encourage them to give their correct address and to report any change of address to health worker staffs and therefore improve adherence to tuberculosis treatment.

The review findings are important for the policy makers, medical practitioners, health worker staff and researchers to study the patient context as a whole (social, behavioral factors, believes and knowledge) and not to ignore their experience and opinion in treatment course. This will make the health care consumer share the responsibility of the treatment process and will combat non-adherence. Moreover, it helps in creating good relationship between the patients, health care providers and the whole community. This new approach in thinking will im- 
prove tuberculosis treatment adherence and will reinforce the surveillance system of tuberculosis control program at state, national and international levels.

In addition to the findings of this systematic review we believe that further studies are needed to be conducted to deeply understand different aspects of tuberculosis disease and its treatment (social and biomedical) aspects which is a complex process. These studies should include the experience of policy makers, the health personnel working in the field, nongovernmental organizations, patient support groups and the community leaders. Moreover, the patients' knowledge about tuberculosis for example, patient satisfaction with health services provision and staff receptiveness. This will substantially help the health care system planners in developing good future strategy to improve treatment adherence among tuberculosis patients.

\section{Conclusions}

Non-adherence to tuberculosis treatment is a complex dynamic process, which was influenced by multiple factors. Identifying and understanding the nature of these factors facilitates development of appropriate and effective intervention plans.

The relationship between the identifiable variables and TB treatment default is important for the knowledge of policy makers and clinicians dealing with TB patients. Despite the fact that association of these variables with increased risk of TB treatment default had been reported in many individual studies, it had not received attention in terms of planning, training, guidelines, and research. This systematic review attempts to alter the current situation with the aim to increase knowledge about treatment default and to promote adherence to TB treatment.

The results of our review can help to find out the consistently statistically related factors to $\mathrm{TB}$ default treatment and assist the health care system designers, health service providers including clinicians, nurses, counselors, patients, families, and the community leading to high adherence to tuberculosis treatment, reinforcing the surveillance system of tuberculosis control program hence achieving the goal of tuberculosis elimination at state, national and international levels.

\section{Acknowledgements}

We would like to thank our Colleagues at MOH Khartoum State and TB control Programme.

\section{Conflicts of Interest}

The authors declare no conflicts of interest regarding the publication of this paper.

\section{References}

[1] World Health Organization (2000) The World Health Report about Health Systems Improving Performance. Geneva.

[2] Murray, C.J. and Lopez, A.D. (1997) Mortality by Cause for Eight Regions of the World: Global Burden of Disease Study. The Lancet, 349, 1269-1276. https://doi.org/10.1016/S0140-6736(96)07493-4 
[3] Jasmin, J., Anju, K. and Renu, B. (2003) Factors Associated with Drug Resistance in Pulmonary Tuberculosis. Indian Journal of Chest and Allied Sciences, 45, 105-109.

[4] Suleiman, M.M.A. and Sodemann, M. (2009) Evaluation of tuberculosis control programme in Khartoum State for the Year 2006. Scandinavian Journal of Public Health, 37, 101-108. https://doi.org/10.1177/1403494808097172

[5] World Health Organization (WHO) (2008) Stop TB Partnership. Tuberculosis Facts. Geneva.

[6] Getahun, H., Gunneberg, C., Granich, R. and Nunn, P. (2010) HIV infection-Associated Tuberculosis: the Epidemiology and the Response. Clinical Infectious Diseases, 50, S201-S207. https://doi.org/10.1086/651492

[7] World Health Organization (WHO) (2008) Global Tuberculosis Control, Surveillance, Planning and Financing 2008.

[8] World Health Organization (WHO) (2004) Guidelines for HIV Surveillance among Tuberculosis Patients. 2nd Edition, Geneva.

[9] World Health Organization (WHO) (2011) Global Tuberculosis Control.

[10] Lönnroth, K., Castro, G.K., Chakaya, J.M., Chauhan, L.S., Floy, K., Glaziou, P. and Raviglione, M.C. (2010) Tuberculosis Control and Elimination 2010-50: Cure, Care, and Social Development. The Lancet, 375, 1814-1829. https://doi.org/10.1016/S0140-6736(10)60483-7

[11] Maher, D. and Raviglione, M. (2005) Global Epidemiology of Tuberculosis. Clinics in Chest Medicine, 26, 167-182. https://doi.org/10.1016/j.ccm.2005.02.009

[12] Kyobutungi, C., Ziraba, A.K., Ezeh, A. and Ye, Y. (2008) The Burden of Disease Profile of Residents of Nairobi's Slums: Results from a Demographic Surveillance System. Population Health Metrics, 6, Article No. 1. https://doi.org/10.1186/1478-7954-6-1

[13] El-Sony, A.I., Khamis, A.H., Enarson, D.A., Baraka, O., Mustafa, S.A. and Bjune, G. (2002) Treatment Results of DOTS in 1797 Sudanese Tuberculosis Patients with or without HIV Co-Infection. International Journal of Tuberculosis and Lung Disease, 6, 1058-1066.

[14] Sloan, J.P. and Sloan, M.C. (1981) An Assessment of Default and Non-Compliance in Tuberculosis Control in Pakistan. Transactions of the Royal Society of Tropical Medicine and Hygiene, 75, 717-718. https://doi.org/10.1016/0035-9203(81)90161-9

[15] Van Der Werf, T.S., Dade, G.K. and Van Der Mark, T.W. (1990) Patient Compliance with Tuberculosis Treatment in Ghana: Factors Influencing Adherence to Therapy in a Rural Service Programme. Tubercle, 71, 247-252. https://doi.org/10.1016/0041-3879(90)90036-8

[16] California Department of Health Services/California Tuberculosis Controllers association Joint Guidelines. Guidelines for the Treatment of Active Tuberculosis Disease.2003.

[17] Chuah, S.Y. (1991) Factors Associated with Poor Patient Compliance with Anti-Tuberculosis Therapy in Northwest Perak, Malaysia. Tubercle; 72, 261-264. https://doi.org/10.1016/0041-3879(91)90051-S

[18] Menzies, R., Rochert, I. and Vissandjee, B. (1993) Factors Associated with Compliance in Treatment of Tuberculosis. Tuber\& and Lung Disease, 74, 32-37. https://doi.org/10.1016/0962-8479(93)90066-7

[19] Wilkinson, D. (1994) High-Compliance Tuberculosis Treatment Programme in a Rural Community. The Lancet, 343, 647-648.

https://doi.org/10.1016/S0140-6736(94)92640-9 
[20] William, J.B., Cohn, D.L., Rietmeijer, C.A., Judson, F.N., Sbarbaro, J.A. and Reves, R.R. (1997) Noncompliance with Directly Observed Therapy for Tuberculosis: Epidemiology and Effect on the Outcome of Treatment. Chest, 111, 1168-1173. https://doi.org/10.1378/chest.111.5.1168

[21] Jasmer, R.M., Seaman, C.B., Gonzalez, L.C., Kawamura, L.M., Osmond, D.H. and Daley, C.L. (2004) Tuberculosis Treatment Outcomes: Directly Observed Therapy Compared with Self-Administered Therapy. American Journal of Respiratory and Critical Care Medicine, 170, 561-566. https://doi.org/10.1164/rccm.200401-095OC

[22] Ngamvithayapong, J., Uthaivoravit, W., Yanai, H., Akarasewi, P. and Sawanpanyalert, P. (1997) Adherence to Tuberculosis Preventive Therapy among HIV-Infected Persons in Chiang Rai, Thailand. AIDS, 11, 107-112. https://doi.org/10.1097/00002030-199701000-00016

[23] World Health Organization () Definitions and Reporting Framework for Tuberculosis-2013 Revision (updated December 2014 and January 2020).

[24] Jochem, K., Fryatt, R.J., Harper, I., White, A., Luitel, H. and Dahal, R. (1997) Tuberculosis Control in Remote Districts of Nepal Comparing Patient-Responsible Short-Course Chemotherapy with Long-Course Treatment. International Journal of Tuberculosis and Lung Disease, 1, 502-508.

[25] Moher, D., Liberati, A., Tetzlaff, J. and Altman, D.G. (2009) Preferred Reporting Items for Systematic Reviews and Meta-Analyses: The PRISMA Statement. BMJ, 339, b2535.

[26] Awofeso, N. (2008) Anti-Tuberculosis Medication Side-Effects Constitute Major Factor for Poor Adherence to Tuberculosis Treatment. Bulletin of the World Health Organization, 86, A-D. https://doi.org/10.2471/BLT.07.043802

[27] Comolet, T.M., Rakotomalala, R. and Rajaonarioa, H. (1998) Factors Determining Compliance with Tuberculosis Treatment in an Urban Environment, Tamatave, Madagascar. International Journal of Tuberculosis and Lung Disease, 11, 891-897.

[28] Kruyt, M.L., Kruyt, N.D., Boeree, M.J., Harries, A.D., Salaniponi, F.M. and van Noord, P.A. (1999) True Status of Smear-Positive Pulmonary Tuberculosis Defaulters in Malawi. Bulletin of the World Health Organization, 77, 386-391.

[29] Edginton, M.E., Sekatane, C.S. and Goldstein, S.J. (2002) Patients' Beliefs: Do They Affect Tuberculosis Control? A Study in a Rural District of South Africa. International Journal of Tuberculosis and Lung Disease, 6, 1075-1082.

[30] Sanou, A., Dembele, M., Theobald, S. and Macq, J. (2004) Access and Adhering to Tuberculosis Treatment: Barriers Faced by Patients and Communities in Burkina Faso. International Journal of Tuberculosis and Lung Disease, 8, 1479-1483.

[31] Chang, K.C., Leung, C.C. and Tam, C.M. (2004) Risk Factors for Defaulting from Anti-Tuberculosis Treatment under Directly Observed Treatment in Hong Kong. International Journal of Tuberculosis and Lung Disease, 8, 1492-1498.

[32] Shargie, E.B. and Lindtjorn, B. (2007) Determinants of Treatment Adherence among Smear-Positive Pulmonary Tuberculosis Patients in Southern Ethiopia. PLoS Medicine, 4, e37. https://doi.org/10.1371/journal.pmed.0040037

[33] Xu, L., Gai, R., Wang, X., Liu, Z., Cheng, J., Zhou, C., et al. (2010) Socio-Economic Factors Affecting the Success of Tuberculosis Treatment in Six Counties of Shandong Province, China. International Journal of Tuberculosis and Lung Disease, 14, 440-446.

[34] Kigozi, G., Heunis, C., Chikobvu, P.C., Botha, S. and Rensburg, D. (2017) Factors Influencing Treatment Default among Tuberculosis Patients in a High Burden Province of South Africa. International Journal of Infectious Diseases, 54, 95-102. https://doi.org/10.1016/j.ijid.2016.11.407 
[35] Alobu, I., Oshi, S.N., Oshi, D.C. and Ukwaja, K.N. (2014) Risk Factors of Treatment Default and Death among Tuberculosis Patients in a Resource-Limited Setting. Asian Pacific Journal of Tropical Medicine, 7, 977-984. https://doi.org/10.1016/S1995-7645(14)60172-3

[36] Abdelhadi, M.A., Talal, E., Mahdi, T.E., Mohammed, A., Soghaier, M.A., Awadalla, H.M., Ahmed, A.E. and Khalid, F.A. (2015) Factors Associated with Default from Treatment among Tuberculosis Patients in Kassala State, Sudan 2013. Journal of Public Health and Epidemiology, 7, 183-188. https://doi.org/10.5897/JPHE2015.0740

[37] Thelma, E.T., Anna, M.C.G. Garfin, Kurbatova E V, Mangan JM, Chi R O, et al. (2016) Factors Associated with Loss to Follow-Up during Treatment for Multidrug-Resistant Tuberculosis, the Philippines, 2012-2014. Emerging Infectious Diseases, 22, 491-502. https://doi.org/10.3201/eid2203.151788

[38] Lackey, B., Seas, C., Stuyft, P.V. and Otero, L. (2015) Patient Characteristics Associated with Tuberculosis Treatment Default: A Cohort Study in a High-Incidence Area of Lima, Peru. PLoS ONE, 10, e0128541. https://doi.org/10.1371/journal.pone.0128541

[39] Slama, K., Tachfouti, N., Obtel, M. and Nejjari, C. (2013) Factors Associated with Treatment Default by Tuberculosis Patients in Fez, Morocco. Eastern Mediterranean Health Journal, 19, 687-693. https://doi.org/10.26719/2013.19.8.687

[40] El-Muttalut, M. and Elnimeiri, M.K. (2017) Factors Contributing to Non-Compliance with Treatment among Tuberculosis Patients, Kassala State, Sudan 2016. International Scholars Journals, 6, 332-338.

[41] Santha, T., Garg, R., Frieden, T.R., Chandrasekaran, V., Subramani, R., Gopi, P.G., et al. (2002) Risk Factors Associated with Default, Failure and Death among Tuberculosis Patients Treated in a DOTS Programme in Tiruvallur District, South India, 2000. International Journal of Tuberculosis and Lung Disease, 6, 780-788.

[42] Hill, P.C., Stevens, W., Hill, S., Bah, J., Donkor, S.A., Jallow, A., et al. (2005) Risk Factors for Defaulting from Tuberculosis Treatment: A Prospective Cohort Study of 301 Cases in The Gambia. International Journal of Tuberculosis and Lung Disease, 9, 1349-1354.

[43] Gelmanova, I.Y., Keshavjee, S., Golubchikova, V.T., Berezina, V.I., Strelis, A.K., Yanova, G.V., et al. (2007) Barriers to Successful Tuberculosis Treatment in Tomsk, Russian Federation: Non-Adherence, Default and the Acquisition of Multidrug Resistance. Bull World Health Organ, 85, 703-711. https://doi.org/10.2471/BLT.06.038331

[44] Talay, F., Kumbetli, S. and Altin, S. (2008) Factors Associated with Treatment Success for Tuberculosis Patients: A Single Center's Experience in Turkey. Japanese Journal of Infectious Diseases, 61, 25-30.

[45] Muture, B., Keraka, M., Kimuu, P., Kabiru, E., Ombeka, V. and Oguya, F. (2011) Factors Associated with Default from Treatment among Tuberculosis Patients in Nairobi Province, Kenya: A Case Control Study. BMC Public Health, 11, Article No. 696. https://doi.org/10.1186/1471-2458-11-696

[46] Finlay, A., Lancaster, J., Holtz, T.H., Weyer, K., Miranda, A. and van der Walt, M. (2012) Patient- and Provider-Level Risk Factors Associated with Default from Tuberculosis Treatment, South Africa, 2002: A Case-Control Study. BMC Public Health, 12, Article No. 56. https://doi.org/10.1186/1471-2458-12-56

[47] Saibannavar, A. and Desai, S. (2016) Risk Factors Associated with Default among Smear Positive TB Patients under RNTCP in Western Maharashtra. Journal of Dental and Medical Sciences, 15, 50-56. 
[48] Cherkaoui, I., Sabouni, R., Ghali, I., Kizub, D., Alexander, C., et al. (2014) Treatment Default amongst Patients with Tuberculosis in Urban Morocco: Predicting and Explaining Default and Post-Default Sputum Smear and Drug Susceptibility Results. PLOS ONE, 9, e93574. https://doi.org/10.1371/journal.pone.0093574

[49] Lalor, M.K., Greig, J., Allamuratova, S., Althomsons, S., Tigay, Z., et al. (2013) Risk Factors Associated with Default from Multi- and Extensively Drug-Resistant Tuberculosis Treatment, Uzbekistan: A Retrospective Cohort Analysis. PLOS ONE, 8, e78364. https://doi.org/10.1371/journal.pone.0078364

[50] Franke, M.F., Appleton, S.C., Bayona, J., Arteaga, F., Palacios, E., Llaro, K., et al. (2008) Risk Factors and Mortality Associated with Default from Multidrug-Resistant Tuberculosis Treatment. Clinical Infectious Diseases, 46, 1844-1851. https://doi.org/10.1086/588292

[51] Kelly, E.D., Ouafae, L., Iraqi, G., Janine, K., Elmessaoud, M.D., Imad, C. and Rajae, E. (2011) Risk Factors for Tuberculosis Treatment Failure, Default, or Relapse and Outcomes of Retreatment in Morocco. BMC Public Health, 11, Article No. 140. https://doi.org/10.1186/1471-2458-11-140

[52] Centers for Disease Control and Prevention MMWR (1998) Acquired Multidrug-Resistant Tuberculosis-Buenaventura, Colombia, 1998. JAMA, 280, 1653. https://doi.org/10.1001/jama.280.19.1653-JWR1118-3-1

[53] Yeung, M.C., Noertjojo, K., Leung, C.C., Chan, S.L. and Tam, C.M. (2003) Prevalence and Predictors of Default from Tuberculosis Treatment in Hong Kong. Hong Kong Med Journal, 9, 263-268.

[54] Vijay, S., Balasangameswara, V.H., Jagannatha, P.S., Saroja, V.N. and Kumar, P. (2003) Defaults among Tuberculosis Patients Treated under DOTS in Bangalore City: A Search for Solution. Indian Journal of Tuberculosis, 50, 185-195.

[55] Roy, N., Basu, M., Das, S., Mandal, A., Dutt, D. and Dasgupta, S. (2015) Risk Factors Associated with Default among Tuberculosis Patients in Darjeeling District of West Bengal, India. Journal of Family Medicine and Primary Care, 4, 388-394. https://doi.org/10.4103/2249-4863.161330

[56] Culqui, D.R., Munayco, E.C.V., Grijalva, C.G., Cayla, J.A., Horna-Campos, O., Alva, Ch.K. and Suarez, O.L.A. (2012) Factors Associated with the Non-Completion of Conventional Anti-Tuberculosis Treatment in Peru. Archivos de Bronconeumología, 48, 150-155. https://doi.org/10.1016/j.arbres.2011.12.008

[57] Ali, A.O.A. and Prins, M.H. (2016) Patient Non Adherence to Tuberculosis Treatment in Sudan: Socio Demographic Factors Influencing Non Adherence to Tuberculosis Therapy in Khartoum State. Pan African Medical Journal, 25, No. 80. https://doi.org/10.11604/pamj.2016.25.80.9447

[58] Ali, A.O.A. and Prins, M.H. (2016) Patient Knowledge and Behavioral Factors Leading to Non-Adherence to Tuberculosis Treatment in Khartoum State, Sudan. Journal of Public Health and Epidemiology, 8, 316-325.

[59] Vasudevan, K., Jayakumar, N. and Gnanasekaran, D. (2014) Smear Conversion, Treatment Outcomes and the Time of Default in Registered Tuberculosis Patients on RNTCP DOTS in Puducherry, Southern India. Journal of Clinical and Diagnostic Research, 8, No. 10. https://doi.org/10.7860/JCDR/2014/8421.4984

[60] Marlucia, S.G., Maria, L.P., Tomàs, M.P., Alexandra, B.S., Leni, S.M., et al. (2012) Factors Associated with Tuberculosis Treatment Default in an Endemic Area of the Brazilian Amazon: A Case Control-Study. PLoS ONE, 7, e39134.

https://doi.org/10.1371/journal.pone.0039134 
[61] Nezenega, Z.S., Gacho, Y.H. and Tafere, T.E. (2013) Patient Satisfaction on Tuberculosis Treatment Service and Adherence to Treatment in Public Health Facilities of Sidama Zone, South Ethiopia. BMC Health Services Research, 13, Article No. 110. https://doi.org/10.1186/1472-6963-13-110

[62] Tanguis, H.G., Cayla, J.A., Garcia de Olalla, P.G., Jansa, J.M. and Brugal, M.T. (2000) Factors Predicting Non-Completion of Tuberculosis Treatment among HIV-Infected Patients in Barcelona (1987-1996). International Journal of Tuberculosis and Lung Disease, 4, 55-60.

[63] Borgdorff, M.W., Veen, J., Kalisvaart, N.A., Broekmans, J.F. and Nagelkerke, N.J.D. (2000) Defaulting from Tuberculosis Treatment in the Netherlands: Rates, Risk Factors and Trend in the Period 1993-1997. European Respiratory Journal, 16, 209-213. https://doi.org/10.1034/j.1399-3003.2000.16b05.x

[64] Daniel, O.J., Oladapo, O.T. and Alausa, O.K. (2006) Default from Tuberculosis Treatment Programme in Sagamu, Nigeria. Nigerian Journal of Medicine, 15, 63-67. https://doi.org/10.4314/njm.v15i1.37119

[65] Kliiman, K. and Altraja, A. (2010) Predictors and Mortality Associated with Treatment Default in Pulmonary Tuberculosis. International Journal of Tuberculosis and Lung Disease, 14, 454-463.

[66] Pablos-Méndez, A., Knirsch, C.A., Barr, R.G., Lerner, B.H. and Frieden, T.R. (1997) Nonadherence in Tuberculosis Treatment: Predictors and Consequences in New York City. The American Journal of Medicine, 102, 164-170. https://doi.org/10.1016/S0002-9343(96)00402-0

[67] Cummings, K.C., Mohle-Boetani, J., Royce, S.E. and Chin, D.P. (1998) Movement of Tuberculosis Patients and the Failure to Complete Anti Tuberculosis Treatment. American Journal of Respiratory and Critical Care Medicine, 157, 1249-1252. https://doi.org/10.1164/ajrccm.157.4.9708058

[68] Uplekar, M., Juvekar, S., Morankar, S., Rangan, S. and Nunn, P. (1998) Tuberculosis Patients and Practitioners in Private Clinics in India. International Journal of Tuberculosis and Lung Disease, 2, 324-329.

[69] Gelaw, M., Genebo, T., Dejene, A., Lemma, E. and Eyob, G. (2001) Attitude and Social Consequences of Tuberculosis in Addis Ababa, Ethiopia. East African Medical Journal, 78, 382-388. https://doi.org/10.4314/eamj.v78i7.9014

[70] Pandit, N. and Choudhary, S.K. (2006) A Study of Treatment Compliance in Directly Observed Therapy for Tuberculosis. Indian Journal of Community Medicine, 31, 241-243.

[71] Kapella, B.K., Anuwatnonthakate, A., Komsakorn, S., Moolphate, S., Charusuntonsri, P., Limsomboon, P., et al. (2009) Directly Observed Treatment Is Associated with Reduced Default among Foreign Tuberculosis Patients in Thailand. International Journal of Tuberculosis and Lung Disease, 13, 232-237.

[72] Samuel, A.B., Tomoko, K., Tomoko, T. and Nobuyuki, H. (2010) Factors Contributing to Tuberculosis (TB) Defaulter Rate in New Juaben Municipality in the Eastern Region of Ghana. Journal of the National Institute of Public Health, 59, 291-297.

[73] Hashim, E.A. and Mohamed, E.Y. (2017) Risk Factors for Default from Tuberculosis (TB) Treatment in Patients Attending a Rural Sudanese Hospital. EC Pulmonology and Respiratory Medicine, 3, 177-182.

[74] Basa, S. and Venkatesh, S. (2015) Study on Default and Its Factors Associated among Tuberculosis Patients Treated under DOTS in Mayurbhanj District, Odisha. Journal of Health Research and Reviews, 2, 25-28. https://doi.org/10.4103/2394-2010.158125 
[75] Ashutosh, T., Girish, A., Sten, H.V., Connie, L.K., Alka, K. and Nalini, S. (2012) Pulmonary Tuberculosis in Mumbai, India: Factors Responsible for Patient and Treatment Delays. International Journal of Preventive Medicine, 3, 569-580.

[76] Tekle, B., Mariam, D.H. and Ali, A. (2002) Defaulting from DOTS and Its Determinants in Three Districts of Arsi Zone in Ethiopia. International Journal of Tuberculosis and Lung Disease, 6, 573-579. 